\title{
HIV-1 Vpr-induced cell death in Schizosaccharomyces pombe is reminiscent of apoptosis
}

Sylvain Huard ${ }^{1,}$, Mingzhong Chen ${ }^{2}$, Kristen E Burdette ${ }^{3}$, Csaba Fenyvuesvolgyi ${ }^{1}$, Min Yu ${ }^{2}$, Robert T Elder ${ }^{2}$, Richard Y Zhao ${ }^{1,2,3,4}$

${ }^{1}$ Department of Pathology, University of Maryland School of Medicine, Baltimore, MD 21201, USA; ${ }^{2}$ Children's Memorial Research Center, Department of Pediatrics, Northwestern University Feinberg School of Medicine, Chicago, IL 60614, USA; ${ }^{3}$ Department of Microbiology and Immunology, University of Maryland School of Medicine, Baltimore, MD 21201, USA; ${ }^{4}$ Institute of Human Virology, University of Maryland School of Medicine, Baltimore, MD 21201, USA

Human immunodeficiency virus type 1 (HIV-1) Vpr induces cell death in mammalian and fission yeast cells, suggesting that Vpr may affect a conserved cellular process. It is unclear, however, whether Vpr-induced yeast cell death mimics Vpr-mediated apoptosis in mammalian cells. We have recently identified a number of Vpr suppressors that not only suppress Vpr-induced cell death in fission yeast, but also block Vpr-induced apoptosis in mammalian cells. These findings suggest that $\mathrm{Vpr}$-induced cell death in yeast may resemble some of the apoptotic processes of mammalian cells. The goal of this study was to develop and validate a fission yeast model system for future studies of apoptosis. Similar to Vpr-induced apoptosis in mammalian cells, we show here that Vpr in fission yeast promotes phosphatidylserine externalization and induces hyperpolarization of mitochondria, leading to changes of mitochondrial membrane potential. Moreover, Vpr triggers production of reactive oxygen species (ROS), indicating that the apoptotic-like cell death might be mediated by ROS. Interestingly, $\mathrm{Vpr}$ induces unique morphologic changes in mitochondria that may provide a simple marker for measuring the apoptotic-like process in fission yeast. To verify this possibility, we tested two Vpr suppressors (EF2 and Hsp16) that suppress Vpr-induced apoptosis in mammalian cells in addition to a newly identified Vpr suppressor (Skp1). All three proteins abolished cell death mediated by Vpr and restored normal mitochondrial morphology in the yeast cells. In conclusion, $\mathrm{Vpr}$-induced cell death in fission yeast resembles the mammalian apoptotic process. Fission yeast may thus potentially be used as a simple model organism for the future study of the apoptotic-like process induced by Vpr and other proapoptotic agents.

Keywords: fission yeast (Schizosaccharomyces pombe), HIV-1 Vpr, apoptosis, mitochondria, model organism Cell Research (2008) 18:961-973. doi: 10.1038/cr.2008.272; published online 12 August 2008

\section{Introduction}

Human immunodeficiency virus type 1 (HIV-1) infection is characterized by the progressive loss of $\mathrm{CD}^{+} \mathrm{T}$-cell lymphocytes that results in loss of immune function and eventual progression to AIDS. The depletion of $\mathrm{CD}^{+}$lymphocytes is believed to be the result of programmed cell

Correspondence: Richard Y Zhao

Tel: +1-410-706-6301; Fax: +1-410-706-6303

E-mail: rzhao@som.umaryland.edu

${ }^{\S}$ Current address: Department of Molecular and Cellular Pharmacology, University of Miami, Miami, FL, USA

Received 13 December 2007; revised 19 February 2008; accepted 12 March 2008; published online 12 August 2008 death or apoptosis of HIV-infected cells [1-3]. A number of studies have indicated that HIV-1 Vpr, a proapoptotic protein, contributes at least in part to $\mathrm{CD} 4^{+}$lymphocyte depletion during viral infection [4-8].

Considerable efforts have been taken to characterize the apoptotic signaling cascade induced by Vpr. However, the mechanism of apoptosis induced by $\mathrm{Vpr}$ is still not fully understood. One model proposes that Vpr induces apoptosis by altering the permeability of the mitochondrial pore $[9,10]$. This mitochondria-dependent pathway is characterized by the depolarization of the mitochondrial membrane potential, which is initiated by mitochondrial outer membrane permeabilization (MOMP) [11]. Vpr is thought to lead to the changes in MOMP by binding to an adenine nucleotide transporter (ANT) protein in the inner 
mitochondrial membrane $[6,7,12]$. Ultimately, Vpr causes the activation of caspase 9 and the release of cytochrome $\mathrm{c}$ to the cytoplasm, leading to apoptosis [13-15].

While there is considerable evidence supporting the idea that Vpr induces apoptosis through changes in MOMP, other studies raise the possibility that Vpr kills cells through other pathways (for a review, see [9]). One report showed that the removal of ANT has no effect on Vprinduced apoptosis, whereas the removal of Bax represses apoptosis [4]. Moreover, Vpr activates caspase 8 [16, 17], which is the main effector of the death receptor-mediated apoptotic pathway. A different report showed that a fragment of $\mathrm{Vpr}$ is capable of inducing cell death without caspase activation [18]. A possible role for the nucleus in Vpr-induced cell death was also suggested when cells with defects in the nuclear pore protein Nup124 became resistant to Vpr-induced cell killing [19]. Together, the results of these studies suggest that mechanisms other than the mitochondria-dependent mechanism may also play a role in Vpr-induced apoptosis.

Unicellular organisms like budding or fission yeast can undergo cell death, with many of the features resembling apoptosis of multicellular organisms [20-22]. For example, some of the dying yeast cells also show mitochondrial depolarization, high levels of reactive oxygen species (ROS), DNA fragmentation, and phosphatidylserine (PS) exposure at the plasma membrane characteristic of apoptotic mammalian cells [23]. Although no yeast homologues of the caspases have thus far been reported, apoptosis-like cellular processes were found in yeast and contribute to the process of programmed cell death $[22,24]$. In many of the cases, increases in ROS, indicating cellular oxidative stress, are associated with apoptosis in yeast and mammalian cells $[22,24]$. ROS production is therefore often considered as an indication of apoptosis, and mitochondria are the main ROS source in ROS-mediated apoptosis [10, 25].

$\mathrm{Vpr}$ has been shown to induce cell death in fission yeast and mammalian cells [26, 27], suggesting that the mechanisms governing these processes must be highly conserved. However, it is unknown whether cell death induced by $\mathrm{Vpr}$ in fission yeast mimics that in mammalian cells. By using fission yeast as an experimental model system, we have previously identified a number of $\mathrm{Vpr}$ suppressors, such as Hsp16, Hsp70, and Ef2, that not only prevent Vpr-induced cell death in fission yeast, but also suppress Vpr-induced apoptosis in mammalian cells [28-31]. These findings suggest that some of the cellular signaling that triggers cell death by $\mathrm{Vpr}$ in fission yeast is similar to the signaling that causes apoptosis in mammalian cells. The goals of this study were to further delineate the cellular process of Vpr-induced cell death in fission yeast and to identify some of the apoptotic markers present in mammalian cells for the development of a new and unique method for studying apoptosis. We hypothesized that $\mathrm{Vpr}$ induces cell death in fission yeast through mechanisms that are similar to and reminiscent of apoptosis in mammalian cells. The results described in this report support this hypothesis. Just as in mammalian cells, Vpr induces cell death in fission yeast via the alteration of mitochondrial organization, changes in the mitochondrial membrane potential, and production of ROS. Furthermore, we found that the changes of mitochondrial morphology induced by Vpr could potentially be used as a unique and simple marker for measuring apoptosis in fission yeast; overexpression of two previously reported suppressors of Vpr-induced apoptosis in mammalian cells not only prevented $\mathrm{Vpr}$-induced cell death but also restored mitochondrial morphology. A new Vpr suppressor is also identified through this strategy.

\section{Results}

Vpr induces cell death and promotes PS externalization in fission yeast cells

Vpr-induced cell death in fission yeast can be demonstrated in several ways. As soon as the vpr gene is expressed, cells stop growing [32]. Staining of vpr-expressing cells with the fluorescent dye FUN-1, which measures intracellular metabolic activity and cell membrane integrity, showed little or no metabolic activity $24 \mathrm{~h}$ after $v p r$ gene induction; cells were metabolically inert by $48 \mathrm{~h}$ [27]. Cell death mediated by Vpr in Schizosaccharomyces pombe can also be demonstrated by the inability of fission yeast cells to form colonies on the $v p r$-inducing agar plates [27,33]. The lack of colony-forming ability thus becomes a simple way to measure Vpr-induced cell death. Figure 1A shows that the fission yeast strain RE007, which carries a single integrated copy of the $v p r$ gene in the chromosome of a wild-type SP223 strain [27, 28], was first grown in selective Edinburgh minimal medium (EMM) until the log phase of growth. The actively growing culture was then plated onto thiamine-containing EMM plates, allowing the cells to repress the expression of $v p r$. Normal-sized colonies were obtained under $v p r$-repressing conditions 3-4 days after incubation at $30^{\circ} \mathrm{C}$ (Figure 1A - left panel). However, no or very tiny colonies were seen when the same cells were plated onto thiamine-free EMM plates that allowed $v p r$ to be expressed (Figure 1A - right panel). Therefore, colony formation can be used as a marker for Vpr-induced cell killing in S. pombe [33].

By using the lack of colony formation as an endpoint for Vpr-induced cell death, we have previously conducted genome-wide screenings of multicopy suppressors of Vprinduced cell death. To search for these Vpr suppressors, an $S$. pombe cDNA expression library was transformed 
A

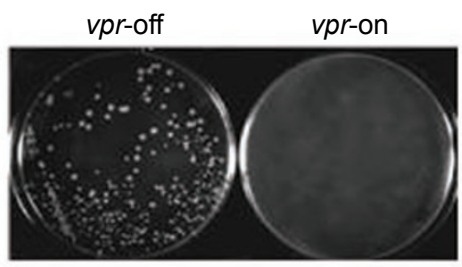

RE007

B

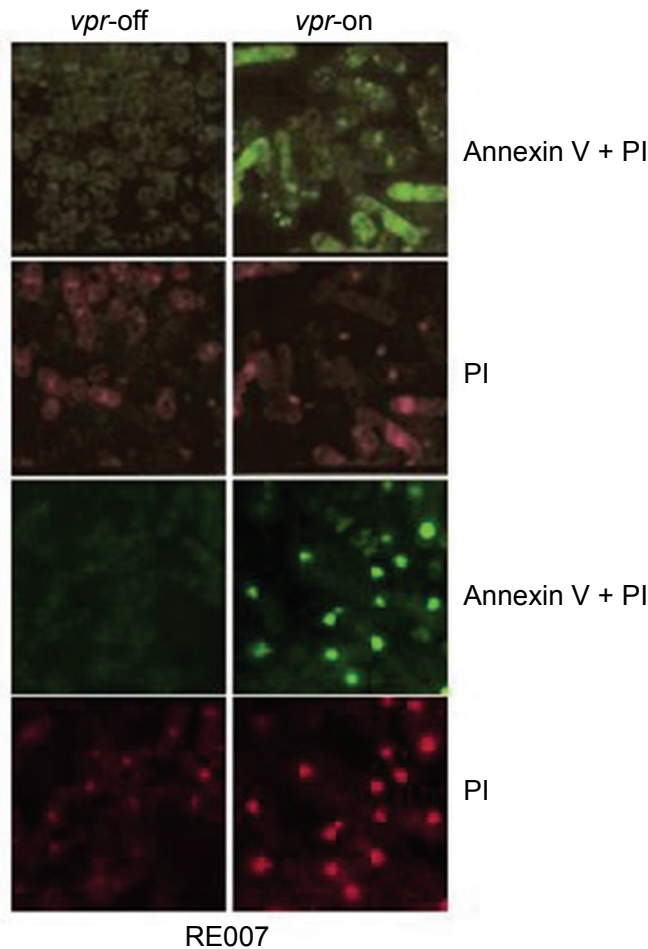

Figure $1 \mathrm{Vpr}$ induces cell death and promotes phosphatidylserine externalization of fission yeast cells. (A) Expression of $v p r$ prevents colony formation of $S$. pombe cells. Vpr induces cell death in $S$. pombe, as shown here by the lack of colony-forming ability onto selective EMM plates under vpr-repressing (vpr-off) and vpr-expressing (vpr-on) conditions [32, 74]. The fission yeast strain RE007, which is a derivative of the wild-type SP223 containing a single integrated copy of $v p r$ gene under the control of the $n m t 1$ promoter [28], was grown in selective EMM to the mid-log growth phase in the presence of thiamine. Equal numbers of cells were streaked onto selective EMM plates with (vpr-off) or without (vpr-on) thiamine. The agar plates were incubated at $30^{\circ} \mathrm{C}$ for 3-4 days before the images were taken. (B) Vpr promotes phosphatidylserine externalization of fission yeast cells as determined by the Annexin V-FITC staining. To visualize phosphatidylserine externalization, the fission yeast strain RE007 was grown in selective EMM in either the presence (vpr-off) or absence (vpr-on) of thiamine to induce $v p r$ gene expression. Cells were collected 32 and $48 \mathrm{~h}$ after gene induction and stained with FITC-labeled Annexin $\mathrm{V}$ and propidium iodine (PI) as previously described [20]. Cells were then visualized microscopically. The PI staining was used here as a control for detecting the integrity of the plasma membrane [22]. Please note that the images shown in the top panels are "Annexin $\mathrm{V}+\mathrm{Pl}$ ", whereas those in the bottom panels are $\mathrm{PI}$ only images. into the RE007 strain [28]. The criterion used to identify suppressors of Vpr-induced cell death was the ability of a fission yeast transformant to form colonies of normal size on the $v p r$-expressing agar plate as previously described (Figure $1 \mathrm{~A} ;[32,33])$. A total of $8.7 \times 10^{5}$ cDNA transformants were screened through three independent screenings. A total of 26 unique sibling groups were identified. Three of them (Hsp16, Hsp70, and Ef2) have been characterized. All of them, when overproduced, prevent Vpr-induced cell death in fission yeast and also suppress Vpr-induced apoptosis in mammalian cells [28-31]. The findings that these suppressors prevent Vpr-induced cell death and apoptosis in both $S$. pombe and mammalian cells raise the interesting possibility that the cellular process resulting in Vpr-induced cell death in fission yeast are in some way similar to those of mammalian cells. To examine this possibility, we first tested possible PS externalization. PS is a membrane phospholipid that typically localizes to the inner layer of the plasma membrane in both prokaryotic and eukaryotic cells [34]. Upon induction of apoptosis, PS redistributes from the inner layer to the outer layer of the membrane, and this redistribution can be detected by Annexin V staining. Therefore, PS externalization detected by Annexin V straining is a sensitive marker for the early stages of apoptosis, shown in both mammalian cells and budding yeast $[20,21]$. For the detection of PS, the RE007 yeast strain was grown under $v p r$-repressing ( $v p r$-off) and $v p r$-expressing ( $v p r$-on) conditions. Cells were treated with zymolyase to remove the cell wall. Spheroplasts were then stained simultaneously with FITC-labeled Annexin V and propidium iodine (PI) to visualize PS exposure and membrane integrity, respectively. As shown in Figure 1B, the $v p r$-repressing control cells showed very weak staining for both FITC-labeled Annexin V and PI (Figure 1B - left panels). However, the majority of the $v p r$-expressing cells at $32 \mathrm{~h}$ after gene induction showed strong green fluorescence in the periphery of the cell, suggesting that some of the PS was exposed at the outer layer of the intact plasma membrane (Figure 1B - top right). To avoid possible artifacts due to the binding of Annexin V to the ruptured plasma membrane, PI, a nuclear DNA dye, was also used to examine the integrity of plasma membrane. The use of this dye is based on the principle that PI cannot pass through an intact membrane [22]. Only few of those Annexin $\mathrm{V}$-stained $v p r$-expressing cells showed red fluorescence in the nucleus, indicating no or little disruption of the plasma membrane of the spheroplasts (Figure 1B - second row). In contrast, relatively strong PI nuclear staining of $v p r$-expressing cells was seen $48 \mathrm{~h}$ after gene induction, indicating PI leakage into the nucleus through a ruptured plasma membrane (Figure 1B, bottom row). Therefore, Vpr-induced cell death in S. pombe displays an apoptotic phenotype as revealed by PS externalization. 


\section{Vpr stimulates the production of ROS}

Upon induction of cellular oxidative stress, large amounts of ROS are released within the cell and often cause cell death $[35,36]$. The major source of ROS production arises from the mitochondria, and mitochondrial dysfunction during cell death enhances ROS production within cells and leads to apoptosis $[37,38]$. To further analyze the role of intracellular ROS in cell death mediated by $\mathrm{Vpr}$, the possible production of ROS was measured upon $v p r$ expression in S. pombe. Dihydroethidium (DHE), a dye capable of indicating the presence of ROS, produces red fluorescence in the presence of ROS and was used for the detection of ROS in our experiment. Expression of $v p r$ was induced in the fission yeast strain RE007, and

A

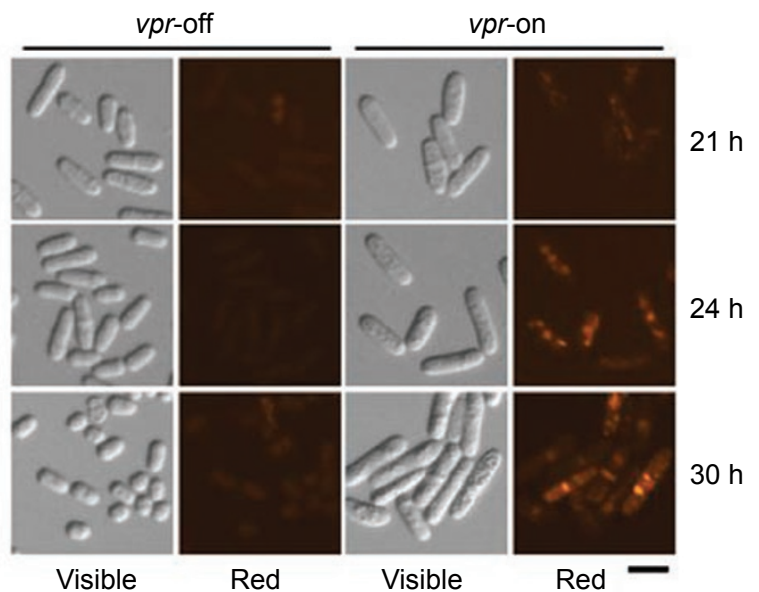

B

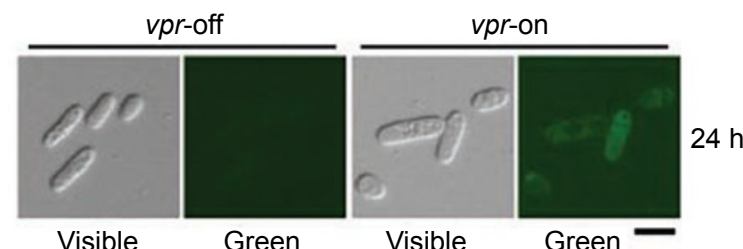

C
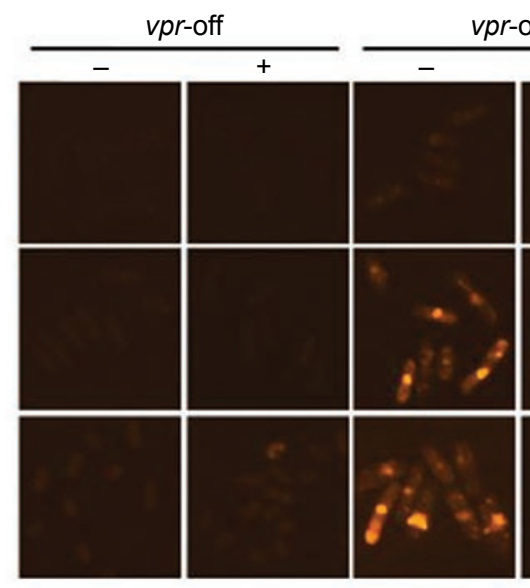

Green
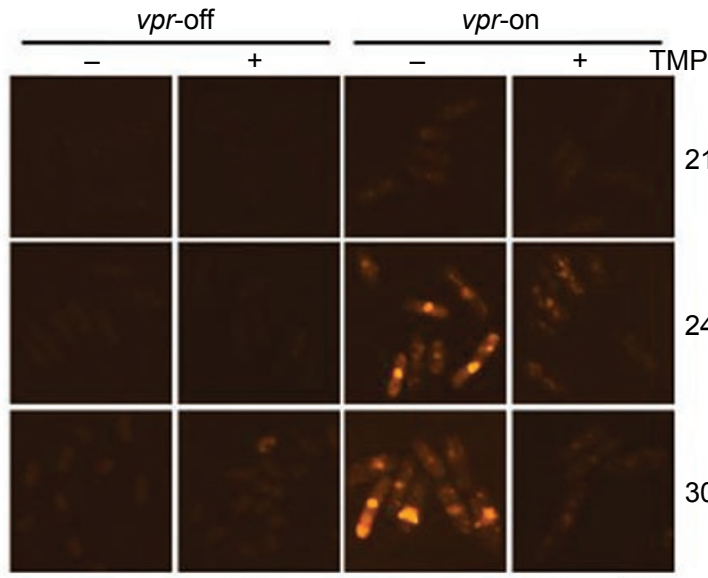

the level of ROS was subsequently detected in cells at 21,24 , and $30 \mathrm{~h}$ after $v p r$ induction (Figure 2A). At $21 \mathrm{~h}$, marginal fluorescence appeared in $v p r$-expressing cells; no significant detectable difference between the $v p r$-repressing and $v p r$-expressing cells was noted (Figure 2A - top row). By $24 \mathrm{~h}$, however, the intensity of the fluorescence in $v p r$-expressing cells became significantly stronger than that in $v p r$-repressing cells (Figure 2A - second row). The intensity of fluorescence greatly increased over time in the $v p r$-expressing cells, whereas the fluorescence remained low in the $v p r$-repressing cells (Figure 2A - bottom row). The increase of ROS production induced by $\mathrm{Vpr}$ was further confirmed by the use of $2^{\prime}, 7^{\prime}$-dichlorodihydrofluorescein diacetate $\left(\mathrm{H}_{2} \mathrm{DCFDA}\right)$. This compound is another ROS dye, which produces green fluorescence in the presence of ROS. Consistent with the DHE staining, the generation of ROS was visualized $24 \mathrm{~h}$ after $v p r$ gene induction. A significant difference in the level of ROS was also observed between $v p r$-repressing and $v p r$-expressing cells (Figure 2B). Therefore, it is clear that $\mathrm{Vpr}$ triggers the production of ROS in S. pombe.

Spin traps, such as 3,3,5,5-tetramethylpyrroline $N$-oxide (TMPO), are compounds that react with free radicals by

Figure $2 \mathrm{Vpr}$ stimulates the production of reactive oxygen species (ROS). (A) Expression of vpr stimulates the production of ROS in $S$. pombe cells. RE007 yeast strain was grown in selective EMM in either the presence (vpr-off) or absence (vpr-on) of thiamine to induce vpr gene induction. $20.5 \mathrm{~h}$ after vpr expression, a ROS indicator dye (DHE) was added to the culture. ROS were detected microscopically over time at the different times indicated. Note that the differences in cell morphology between vpr-off and vpr-on cells are due to the cell cycle arrest induced by Vpr in the vpr-on cells. This phenotype is not directly relevant to ROS production. (B) As an alternative procedure, ROS was also visualized microscopically with a different ROS dye ( $\left.\mathrm{H}_{2} \mathrm{DCFDA}\right)$, which was added to the culture $22.5 \mathrm{~h}$ after vpr expression and detected at $24 \mathrm{~h}$. Note that the staining patterns of DHE and $\mathrm{H}_{2}$ DCFDA are different. Even though both the DHE and $\mathrm{H}_{2}$ DCFDA detect ROS production, DHE is selectively oxidized by superoxide anion whereas fluorescence of $\mathrm{H}_{2}$ DCFDA is triggered by oxidation via hydroxides, hydrogen peroxides, and hydroxyl radicals $[75,76]$. Since the mitochondria are a major source of superoxide, stronger mitochondrial fluorescence was seen with DHE than $\mathrm{H}_{2}$ DCFDA. (C) Treatment of vpr-expressing cells with oxygen radical scavengers (TMPO) reduces ROS accumulation. The RE007 yeast strain was grown in selective EMM in either the presence (vpr-off) or absence (vpr-on) of thiamine to induce vpr gene induction. TMPO was added twice during the course of vpr expression. TMPO was first added to the culture at the time of cell inoculation when vpr gene expression was induced, and cells were treated again with TMPO when DHE was added to the culture to visualize ROS under the microscope at the different times indicated. Scale bar: $10 \mu \mathrm{m}$. 
forming stable complexes. These compounds can therefore be used as ROS scavengers to remove free radical species [39]. If Vpr stimulates ROS production, treatment with a spin trap reagent should decrease or reverse the levels of ROS present in $v p r$-expressing cells. Upon $v p r$ expression, TMPO was added to the RE007 culture. The level of ROS was subsequently detected by DHE at 21, 24, and $30 \mathrm{~h}$ after $v p r$ induction (Figure 2C). Insignificant levels of fluorescence were observed in $v p r$-repressing cells either treated or not treated with TMPO (Figure 2C - first and second columns). A significant reduction of the intensity of fluorescence was detected in the TMPO-treated $v p r-$ expressing cells in comparison with $v p r$-expressing cells that were not treated with TMPO (Figure 2C - third and last columns). However, the amount of ROS in TMPOtreated $v p r$-expressing cells was still higher than that in $v p r$-repressing cells. Therefore, treatment of $v p r$-expressing cells with TMPO only partially relieves the ROS production induced by Vpr.

Vpr alters mitochondrial membrane potential, morphology, and distribution

In mammalian cells, Vpr-induced apoptosis depends to a large extent on the ability to modulate mitochondrial functions $[6,7,18]$. For example, Vpr triggers apoptosis through a pathway that affects mitochondrial membrane permeabilization and leads to changes of the membrane potential $[6$, $7,18]$. To test whether $\mathrm{Vpr}$ also affects mitochondrial membrane potential in $S$. pombe, the mitochondrial membrane potential $\left(\triangle \psi_{\mathrm{m}}\right)$ was assessed in $S$. pombe expressing $v p r$. Expression of $v p r$ was induced in the fission yeast strain RE007, and the yeast $\triangle \psi_{\mathrm{m}}$ was measured by using the $\triangle \psi_{\mathrm{m}}$-sensitive dye 3,3'-dihexyloxacarbocyanine iodide $\left(\mathrm{DiOC}_{6}(3)\right)$ [40]. As shown in Figure 3A, expression of $v p r$ resulted in an increase in $\triangle \psi_{\mathrm{m}}$, as indicated by the shift of the $\mathrm{DiOC}_{6}(3)$ peak to the right. Therefore, it appears that Vpr does, indeed, affect mitochondrial $\triangle \psi_{\mathrm{m}}$ and leads to hyperpolarization of the mitochondria.

Our previous studies suggested that Vpr-induced cell death in $S$. pombe was due in part to the interruption of cytoskeletal structures and intracellular structural proteins like actin [27]. Here, we were interested in whether Vpr has any effect on mitochondrial morphology and organization in $S$. pombe. The fission yeast strains RE007 and MZ01 were used to measure the possible dosage effects of $\mathrm{Vpr}$ on mitochondria by expressing $v p r$ through either an autonomous multicopy pYZ1N-vpr plasmid (MZ01) [41] or a single copy vpr gene (RE007) [28]. To visualize mitochondrial morphology and distribution, fission yeast cells were stained with the mitochondria-specific dye 2-(4-dimethylaminostyryl)-1-methylpyridinium iodide (DASPMI) [42]. Consistent with the previous description

\section{A}

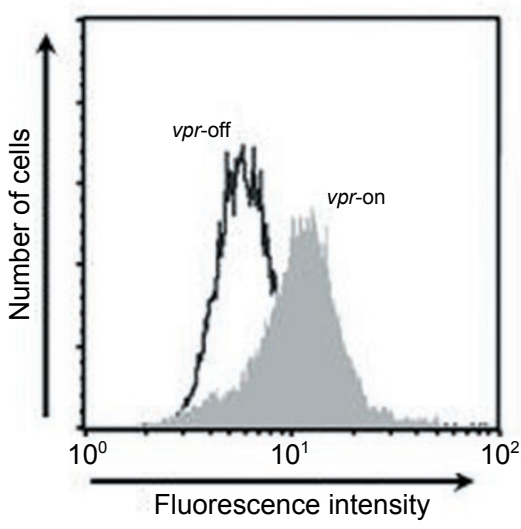

B
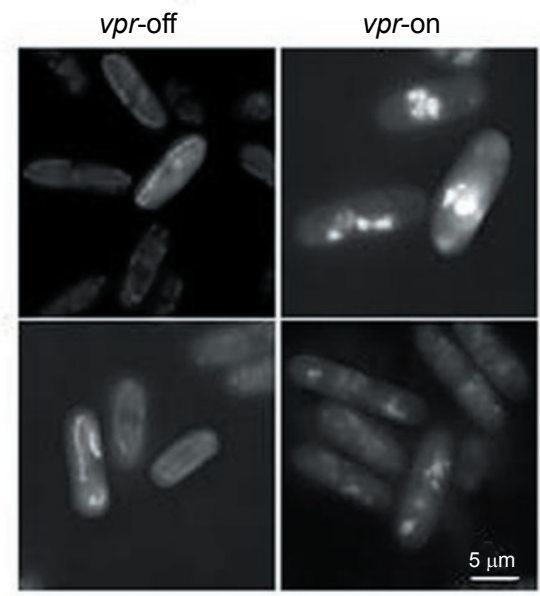

RE007

MZ01

Figure $3 \mathrm{Vpr}$ alters mitochondrial morphology, distribution, and membrane potential. (A) Expression of $v p r$ alters mitochondrial membrane potential $\left(\triangle \psi_{\mathrm{m}}\right)$ and results in the hyperpolarization of mitochondria in S. pombe. The RE007 yeast strain was grown in selective EMM in either the presence (vpr-off) or absence (vpr-on) of thiamine to induce vpr gene induction. Cells were collected $24 \mathrm{~h}$ after gene induction and stained with a $\triangle \psi_{\mathrm{m}}$-sensitive dye $\mathrm{DiOC}_{6}(3)$. Cells were then subjected to FACScan analysis to determine the $\triangle \psi_{m}$ variation. (B) Expression of $v p r$ alters mitochondrial morphology and distribution. Fission yeast wildtype strains (SP223) containing multiple copies (MZ01, in which the $v p r$ gene is expressed episomally from a pYZ1N plasmid) or a single copy (RE007, in which the vpr gene was expressed through a single integrated copy of vpr gene in the chromosome) of $\mathrm{Vpr}$ were grown in selective EMM in either the presence ( $v p r$ off) or absence (vpr-on) of thiamine to induce vprgene induction. Mitochondrial morphologies were visualized microscopically $24 \mathrm{~h}$ after gene induction by staining $S$. pombe cells with DASPMI as previously described [43].

of a normal fission yeast mitochondria [43], mitochondria in the $v p r$-repressing cells appeared either as multiple small dots concentrated around the edge and at the growing ends of the cell, or as a tubular network extending along the 


\section{SP223 + pYZ1N-vpr}

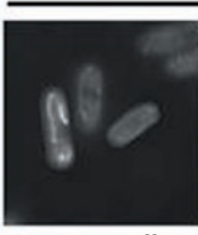

WT-off

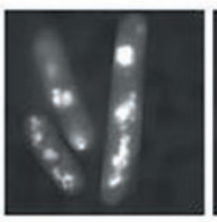

E24G

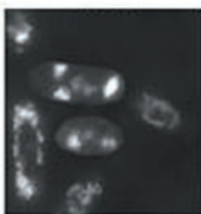

F34I

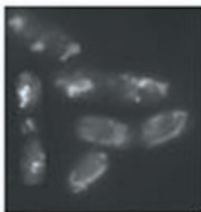

S79A

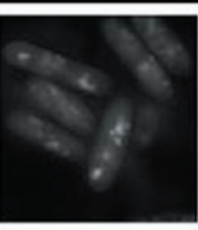

WT-on

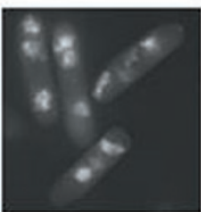

E25K

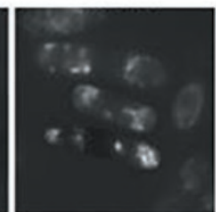

W54R

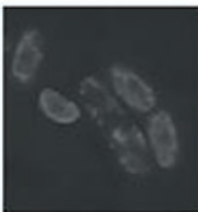

R88K

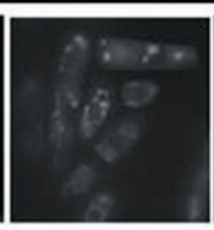

E17D

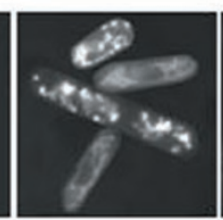

K27A

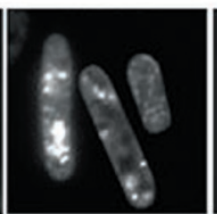

$\mathrm{H} 71 \mathrm{R}$

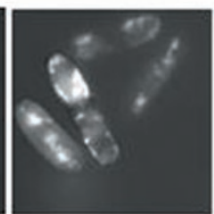

A89T

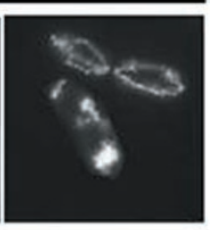

W18R

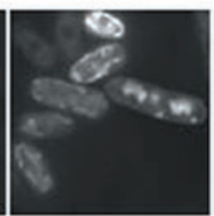

$\mathrm{H} 33 \mathrm{R}$

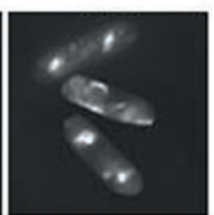

$\mathrm{H} 78 \mathrm{R}$

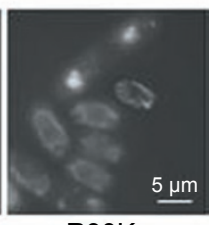

R90K
Figure 4 Correlation of Vpr-induced cell death with alternations of mitochondrial morphology caused by $\mathrm{Vpr}$. A panel of mutated Vpr genes, which were previously described for their abilities to induce cell death in fission yeast cells [33], were used in this study to evaluate their effects on mitochondrial morphology in wild-type SP223 cells. Staining of S. pombe cells with DASPMI as previously described in Figure 3 shows that mitochondrial morphology was affected by these Vpr mutants $24 \mathrm{~h}$ after gene induction. Vpr-induced changes of mitochondrial morphology appear to correlate with Vpr-induced cell death, and this correlation is summarized in Table 1.

periphery of the cell (Figure 3B, left panels). In contrast, different sizes of mitochondrial aggregates were situated near the center of the $v p r$-expressing cells (Figure 3B, right panels). It appears that low levels of $\mathrm{Vpr}$ are sufficient to induce morphologic changes in mitochondria, because similar mitochondrial aggregates were seen in cells expressing single or multiple copies of $v p r$ genes (Figure 3B, right panels). Vpr-induced changes of mitochondrial membrane potential were further confirmed through the use of a different mitochondrial dye provided in a commercial Mitotracker assay (data not shown; Molecular Probes, Eugene, OR). Therefore, Vpr alters mitochondrial morphology and distribution in $S$. pombe cells.

Mutational analysis and correlation of Vpr-induced cell death with changes of mitochondrial morphology

Since Vpr affects mitochondrial morphology and membrane potential, we reasoned that the mitochondrial morphologic changes caused by Vpr may contribute at least in part to Vpr-induced cell death. If this were true, changes of mitochondrial morphology might provide an additional simple marker for measuring Vpr-induced cell death and predicting Vpr-induced apoptosis. To further explore this possibility, the potential correlation between Vpr-induced cell death and mitochondrial morphologic changes was tested. We carried out a mutational analysis using a Vpr mutant panel that has been described previously for Vprinduced cell death in fission yeast [33]. This Vpr mutant

Table 1 Mutant effects of Vpr on mitochondrial morphology and their correlation with Vpr-induced cell death

\begin{tabular}{|c|c|c|c|}
\hline Vpr genotype & $\begin{array}{l}\text { Structural } \\
\text { domain }^{1}\end{array}$ & Cell killing $^{2}$ & $\begin{array}{l}\text { Mitochondrial } \\
\text { morphologic } \\
\text { changes }^{3}\end{array}$ \\
\hline No Vpr & $\mathrm{n} / \mathrm{a}$ & - & --- \\
\hline Wt Vpr & Full length & +++ & +++ \\
\hline \multicolumn{4}{|l|}{ Vpr mutants ${ }^{4}$} \\
\hline E17D & $\alpha 1$ & +++ & $+/--?$ \\
\hline W18R & $\alpha 1$ & + & $+1--$ \\
\hline $\mathrm{E} 24 \mathrm{G}$ & $\alpha 1$ & ++ & +++ \\
\hline E25K & $\alpha 1$ & $+++^{5}$ & +++ \\
\hline $\mathrm{K} 27 \mathrm{~A}$ & $\alpha 1$ & ND & $+/-$ \\
\hline H33R & $\alpha 1$ & ++ & $+1--$ \\
\hline F34I & $\alpha 1$ & + & $+/--$ \\
\hline W54R & $\alpha 2$ & ++ & ++- \\
\hline H71R & $\alpha 2$ & ++ & +++ \\
\hline H78R & $\alpha 2$ & +++ & +++ \\
\hline S79A & $\mathrm{C}$ tail & +++ & $++/-$ \\
\hline $\mathrm{R} 88 \mathrm{~K}$ & $\mathrm{C}$ tail & +++ & $---?$ \\
\hline A89T & $\mathrm{C}$ tail & +++ & +++ \\
\hline R90K & $\mathrm{C}$ tail & +++ & $+/--?$ \\
\hline
\end{tabular}

n/a, not applicable; wt, wild type; ND, not determined.

${ }^{1}$ The structural domains of Vpr affected by these mutants are based on previous reports [44, 45]: $\alpha 1, \alpha$-helix from aa 17-46; $\alpha 2, \alpha$-helix from aa 53-78; C tail, aa 79-96.

${ }^{2}$ The abilities of these Vpr mutants to kill fission yeast cells have been described previously [33]. The levels of cell killing were defined as: +++ , $>80 \%$; ++, 30-79\%; +, 10-29\%; - , < 10\% cell death.

${ }^{3}$ The levels of mitochondrial morphologic changes were determined by the proportion of normal mitochondria (-) vs abnormal mitochondria $(+)$ in a particular Vpr mutant.

${ }^{4}$ All Vpr mutants except K27A were generated by Selig et al. [77].

${ }^{5}$ An early study mistakenly reported that E25K showed no cell-killing effect.

The "?" marker indicates the lack of correlation between Vpr-induced cell killing and mitochondrial morphology. 
A

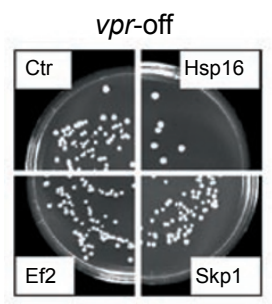

B

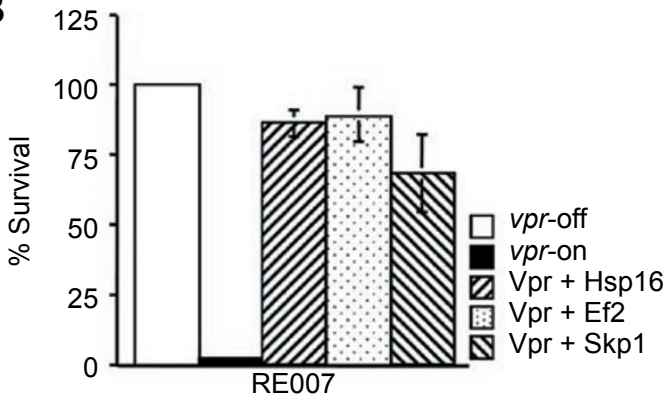

C

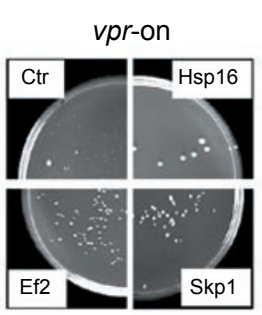

vpr-on

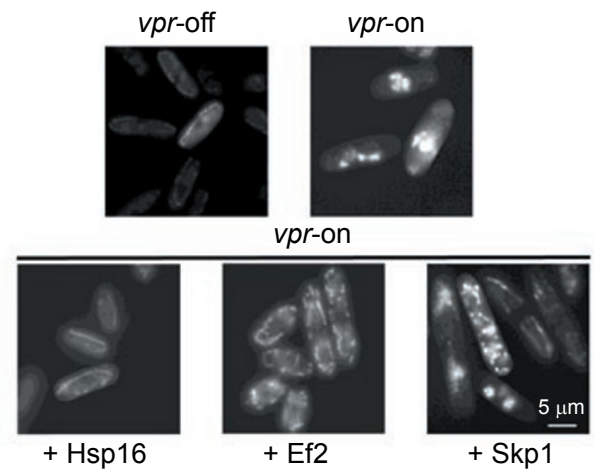

Figure 5 Suppression of Vpr-induced cell death and mitochondrial morphologic changes by Hsp16, Ef2, and Skp1. Ef2 and Hsp16 have been previously shown to suppress Vpr-induced apoptosis in mammalian cells [28, 29, 31]. Skp1 is another Vpr suppressor identified through our prior genome-wide screenings of fission yeast. (A) Expression of hsp16, ef2 and skp1 suppress cell death mediated by $\mathrm{Vpr}$ as shown by their abilities to re-establish the colony-forming ability on the selective EMM plates under the vpr-expressing (vpr-on) conditions. A RE007 yeast strain transformed with pYZ1N, pYZ1N-hsp16, pYZ1N-ef2 or pYZ1N-skp1 was grown in selective EMM to mid-log growth phase in the presence of thiamine. Equal number of cells was plated onto selective EMM plates with (vpr-off) or without (vpr-on) thiamine. Agar plates were incubated at $30^{\circ} \mathrm{C}$ for $3-4$ days. (B) Quantitative measurement of suppression of Vpr-induced cell death by Hsp16, Ef2 and Skp1. Cell survival in the vpr-expressing RE007 cells was determined $24 \mathrm{~h}$ after gene induction at $30^{\circ} \mathrm{C}$ by the colony forming ability on agar plates as described previously [27]. Cell survival was measured as the \% of colonies grown on the vpr-expressing agar plates in comparison with the number of colonies formed on the vpr-repressing agar plates. Cell survival of the vpr-off cultures was adjusted to $100 \%$ for comparison purposes. Data presented in the figure represent mean $\pm S D$ of three experiments. (C) Expression of $h s p 16$, ef2 and skp1 restore morphology and normal distribution of mitochondria in the vpr-expressing RE007 cells. RE007 yeast strain transformed with pYZ1N-hsp16, pYZ1N-ef2 or pYZ1N-skp1 was grown in selective EMM either in the presence (gene-off) or absence (gene-on) of thiamine to induce vpr and suppressor gene expressions. Mitochondrial morphology and distribution were visualized microscopically 24 hours after gene induction by staining S. pombe cells with DASPMI. Scale bar: $5 \mu \mathrm{m}$.

panel includes $14 v p r$ constructs containing single-point mutations representing single amino acid substitutions distributed throughout the entire protein. The structural domain of $\mathrm{Vpr}$ affected by these mutants is summarized in Table 1 and is based on previous reports $[44,45]$. The $\mathrm{Vpr}$ mutants were cloned into a fission yeast expression vector pYZ1N [41]. Each of the plasmids that carry a specific $v p r$ gene mutation was transformed into the wild-type SP223 fission yeast strain, and the effect of Vpr on mitochondrial morphology was examined as described in Figure 3B. DNA sequencing and immunoblot analysis confirmed that all of the $v p r$ mutations were expressed properly in fission yeast [33]. Thus, any changes of mitochondrial morphology in these $\mathrm{Vpr}$ point mutation experiments are due to the specific Vpr mutation. The levels of mitochondrial morphologic changes were determined by the numbers of cells containing abnormal mitochondria (marked as "+") in comparison with the numbers of cells carrying normal mitochondria (marked as "-") for a cell population carrying a particular Vpr mutant. As shown in Figure 4, Vpr-induced changes of mitochondrial morphology appear to a large extent to correlate with Vpr-induced cell death; this correlation is summarized in Table 1. For example, the maximum levels of cell death $(>80 \%)$ induced by the E25K, H78R, and A89T Vpr mutants [33] correlate with the most profound changes of mitochondrial morphology. Significant mitochondrial morphology changes were also associated with the E24G and H71R mutants, which induced intermediate levels (30-79\%) of cell death; the minimal levels of Vprinduced cell death (10-29\%) conferred by the W18R and 
F34I mutants were associated with significantly reduced changes of mitochondrial morphology (Figure 4). However, discordance was also noticed. Even though expression of the E17D, R88K, or R90K mutants caused significant cell death, little (E17D and R90K) or no (R88K) mitochondrial morphologic changes were detected in cells expressing these mutants. These observations may suggest that cell death induced by the three Vpr mutants (E17D, R88K, and R90K) occurs through mechanisms other than the mitochondria-dependent mechanism proposed above. These possibilities will be further explained in the Discussion section.

Overexpression of apoptotic suppressors of Hsp16 and Ef2 ameliorates cell death and mitochondrial morphology changes induced by $\mathrm{Vpr}$

We show above that Vpr-induced cell death in fission yeast resembles the apoptotic process observed in mammalian cells. This suggests the interesting possibility that fission yeast may serve as a simple model organism for studying apoptosis. Further, yeast may potentially be used for large-scale screening of anti-apoptotic factors. Indeed, through genome-wide screenings of multicopy suppressors of Vpr-induced cell death in fission yeast, we have identified several fission yeast proteins (e.g. Hsp16, Hsp70, and Ef2) that can specifically block Vpr-induced apoptosis in mammalian cells [28-31]. As shown in Figure 5A, Vpr strongly inhibited colony formation under $v p r$-expressing conditions (Figure 5A - top left). When hsp16, ef2, or skpl was co-expressed with $v p r$, however, cells regained their ability to form colonies under $v p r$-expressing conditions (Figure 5A). Co-expression of hspl6 or ef 2 with $v p r$ restored cell survival to about $80-90 \%$; expression of the Vpr suppressor $s k p 1$, which was identified through the same genome-wide screenings as Hsp16, revitalized about 70\% of the $v p r$-expressing cells (Figure 5B). Therefore, these specific suppressors prevent cell death mediated by Vpr.

If fission yeast could be used as a simple model system to screen for anti-apoptotic factors, the colony-forming ability of yeast cells could be used as the primary indicative marker for cell survival. It would be even better if another specific, mitochondria-dependent endpoint could also be used as an alternative simple marker. Measuring mitochondrial morphology offers an attractive assay due to its simplicity. To test this possibility, we were interested in seeing whether overexpression of $h s p 16$, ef 2 , and $s k p 1$ was also capable of correcting the aberrant organization of mitochondria induced by Vpr. As shown in Figure 5C, $v p r$ gene expression in the RE007 cells induced formation of large mitochondrial aggregates (Figure 5C - top right). However, co-expression of $h s p 16$ or ef2 with $v p r$ restored the normal mitochondrial morphology and distribution, which are characterized by multiple small dots and tubular networks localized at the periphery of the cell (Figure 5C - top left and second row). Consistent with the incomplete suppressive activity of Skp1 toward Vpr, expression of skp 1 partially restored the normal organization of mitochondria within the $v p r$-expressing cells (Figure 5C - bottom row). Therefore, overproduction of apoptotic suppressors of Hsp16 or Ef2 not only prevented Vpr-induced cell death, but also corrected the mitochondrial abnormalities caused by Vpr. Skp1, a Vpr suppressor identified from a prior yeast screening, also has suppressive activity against the cell death and mitochondrial abnormalities induced by Vpr.

\section{Discussion}

We demonstrated here that Vpr-induced cell death in fission yeast resembles many aspects of mammalian apoptotic-like cellular processes. To our knowledge, this is the first report that systematically describes the pro-apoptotic effects of $\mathrm{Vpr}$ in fission yeast in comparison with its effects in mammalian cells. Similar to the ramification of mammalian apoptosis, Vpr promotes both PS externalization and the production of ROS in fission yeast cells (Figures 1 and 2); Vpr alters mitochondrial membrane potentials and produces accompanying changes of mitochondrial morphology and distribution within fission yeast cells (Figure 3). These findings suggest that fission yeast may offer a simple alternative model for the study of apoptosis generated through the mitochondria-dependent mechanism. We further suggest that the change of mitochondrial morphology could potentially be used as a marker for testing the apoptotic-like processes in fission yeast. This proposition is further supported by our proof-of-concept studies, showing that the overexpression of Vpr suppressors ef 2 and $h s p 16$, both of which suppress Vpr-induced apoptosis in mammalian cells $[28,31]$, not only prevent $\mathrm{Vpr}$-induced cell death in $S$. pombe but also restore normal mitochondrial morphology and distribution (Figure 5).

Although it is still unclear whether budding or fission yeast offers a reliable model system for the study of apoptosis, apoptotic-like processes have been documented numerous times in both types of yeast [22, 24, 46, 47]. For instance, the expression of mammalian pro-apoptotic genes like Bak, Bax, and $\mathrm{p} 53$ in S. pombe and S. cerevisiae causes cell death [48-51]. This apoptotic-like cell death in yeast is characterized by such typical apoptotic features as chromatin condensation, DNA cleavage, PS exposure, and cytochrome $c$ release from mitochondria $[22,52,53]$. The fact that Vpr triggers cell death in a manner that is reminiscent of apoptosis, as also shown in this study, provides an additional piece of evidence supporting fission yeast as a plausible model for the study of apoptosis. 
PS externalization is a sensitive marker for the early stages of apoptosis in both mammalian cells and budding yeast [34]. We show here that Vpr induces PS externalization in fission yeast, indicating the activation of a similar apoptotic signal. Externalization of PS as a result of apoptosis in mammalian cells is thought to be the signal by which apoptotic cells are recognized for phagocytosis [34]. However, the biological significance of PS externalization in yeast is not fully understood. In mammalian cells, PS is synthesized in the $\mathrm{ER}$ and/or mitochondria-associated membranes. A similar pathway appears to operate in yeast [34]. Therefore, Vprinduced PS externalization in fission yeast may resemble a similar signaling process that triggers apoptosis in mammalian cells. Further investigation is certainly warranted to verify this finding.

Vpr-induced apoptosis is closely associated with the function of mitochondria, especially with mitochondrial membrane permeabilization in both mammalian cells and budding yeast (for reviews, see [9, 10, 54]). The first implication for a mitochondrial role in the Vpr effect actually arose from budding yeast. Macreadie et al. [55] found that expression of $v p r$ at intermediate levels prevented cell growth on nonfermentable carbon sources like glycerol. Since yeast with inactive mitochondria are unable to grow on glycerol but can grow on glucose, these observations indicated that $\mathrm{Vpr}$ inactivates yeast mitochondria. Subsequently, Jacotot et al. [7] presented direct evidence describing the role of MMP in Vpr-induced apoptosis in both human and yeast cells. It is now clear that Vpr induces mitochondria-dependent apoptosis through direct interaction with the permeability transition pore complex (PTPC), where Vpr cooperates with the ANT to form large conductance channels and trigger change of the MMP. Thus, the induction of MMP through interaction with the PTPC appears to be a major mechanism for cell death induced by Vpr in mammalian and yeast cells. Consistent with these prior studies, we now show that Vpr affects mitochondrial membrane potential in fission yeast (Figure $3 \mathrm{~A}$ ). However, it is currently unclear whether $\mathrm{Vpr}$ induces changes of mitochondrial membrane potential by the same mechanism in mammalian and budding yeast cells. It is notable that we show here that Vpr induces hyperpolarization of mitochondrial membrane potential in fission yeast. In previous studies, however, Vpr peptide has been shown to induce apoptosis through the induction of mitochondrial depolarization following binding to the ANT $[6,7]$. A number of previous studies have demonstrated that both mitochondrial hyperpolarization and depolarization contribute to cytochrome $c$ release and apoptosis. In fact, an initial rise in $\triangle \psi$ typically occurs before or simultaneously with cytochrome $c$ release, preceding caspase activation and subsequent membrane depolarization [56-61]. Therefore, a plausible explanation suggests that an early effect of $\mathrm{Vpr}$ on the membrane potential was observed here in fission yeast and that the subsequent later event was detected in previous studies $[6,7]$.

The production of ROS plays a central role in mediating apoptosis in mammalian cells [62] and apoptotic-like cell death in yeast $[22,24]$. Moreover, ROS-mediated apoptosis often correlates with the disruption of mitochondrial membrane potential $[63,64]$. Thus the specific role of ROS in Vpr-induced apoptosis remains unclear. However, Vpr-mediated ROS production has not been reported in mammalian cells. We show here that Vpr promotes the production of ROS (Figure 2A, 2B), suggesting a possible link between Vpr-induced apoptosis and ROS. Noticeably, treatment of $v p r$-expressing cells with the use of oxygen radical scavengers (TMPO) did not completely block Vprinduced cell death as measured by colony-forming ability (data not shown). This observation indicates that a very low level of ROS may have been sufficient to cause cell death and mitochondrial changes, since TMPO did not completely abolish ROS production (Figure 2C). Alternatively, the generation of ROS may not be essential for inducing cell death, but it may rather act as a byproduct of the induction of apoptosis.

Prior studies in our laboratory and others have demonstrated that Vpr also kills budding and fission yeast, which exhibit some of the other characteristics expected for apoptotic cells in the process [27, 32, 55]. For example, one strong indication of fission yeast cell death after expression of $v p r$ is the condensation of the chromatin around the periphery of the nucleus; such condensation is also seen in the early stages of apoptosis in mammalian cells [27, 65].

Altogether, the results of earlier studies together with ours provide strong evidence suggesting that $\mathrm{Vpr}$-induced cell death in $S$. pombe resembles in many ways apoptosis caused by $\mathrm{Vpr}$ in mammalian cells. This strong similarity between fission yeast and mammalian cells in terms of the effects of Vpr on cell death supports the potential use of fission yeast as a model organism for studying apoptosis induced by Vpror other similar apoptosis-inducing agents. The obvious advantage of using such a simple single-celled organism to study apoptosis arises from its potential for large-scale screening of pro-apoptotic or anti-apoptotic agents. The colony-forming ability or the inability of the fission yeast cells to grow could be used as a primary endpoint for initial screenings. However, an alternative and more specific endpoint will also be needed to further verify the initial findings before the factors of interest are confirmed in mammalian cells. We propose that the changes in mitochondrial morphology could serve as this simple alternative endpoint, especially for measuring apoptosis induced through the mitochondria-dependent mechanism. 
Indeed, we have demonstrated as a proof-of-concept that the overexpression of Ef2 and Hsp16, two Vpr suppressors previously shown to suppress $\mathrm{Vpr}$-induced apoptosis in mammalian cells $[28,29,31]$, not only restored the colonyforming abilities of the $v p r$-expressing cells (Figure 5A) but also re-established the normal morphology and distribution of mitochondria in those cells (Figure 5C).

Our previous mutagenesis studies of Vpr-induced cell death indicated that point mutations throughout the entire Vpr protein sequence affected the gene's ability to kill cells [33], suggesting that a large part of the Vpr protein is required for its cell-killing capacity. Consistent with this notion, positive correlations between Vpr-induced cell death and mitochondrial morphologic changes were also found in most of the Vpr mutants (Figure 4; Table 1). However, discordance was observed in three of the point mutations (E17D, R88K, and R90K). The underlying reason for these inconsistent results is currently unclear. One possibility is that Vpr has been shown to induce cell death via both mitochondria-dependent and mitochondria-independent pathways $[27,66]$. In addition to the mitochondria-dependent mechanism described in this manuscript, other cell death mechanisms like necrosis or the interruption of the nuclear membrane could also contribute to Vpr-induced cell death $[19,27]$. Since cell death induced by Vpr could be mediated through other mechanisms involving mitochondria-independent pathways or necrosis [9], it is possible that the strong cell death induced by these Vpr mutants is independent of the mitochondria. The R88K mutant is of particular interest: although it is lethal $(90.5 \pm 2.7 \%$ of cell killing) to fission yeast [33], no obvious changes in mitochondrial morphology were observed in cells expressing this mutant (Figure 4).

By using both colony-forming ability and changes of mitochondrial morphology as endpoints for screening suppressors of Vpr-induced cell death in fission yeast cells, we have identified Skp1 as a new suppressor for Vpr-induced cell death (Figure 5). Similar to Hsp16, Hsp70, and Ef2, Skp1 was initially identified as a possible Vpr suppressor through a genome-wide screen of a fission yeast cDNA library. Based on protein sequence alignment, fission yeast Skp1 is most likely the homologue of mammalian glycogen synthase kinase-3 (GSK3). Roughly $60-64 \%$ of the protein sequences are identical, and $75-78 \%$ of the amino acids are positively correlated over the entire proteins. GSK3 is a member of a highly conserved family of protein serine/threonine kinases that are involved in the regulation of a diverse array of cellular functions, including protein synthesis, cell proliferation and differentiation, microtubule assembly/disassembly, and apoptosis (for reviews, see $[67,68])$. However, the specific role of Skp1/GSK3 in apoptosis is unclear and complex [69]. In fact, GSK3 ap- pears to play both pro- and anti-apoptotic regulatory roles. GSK3 promotes mitochondria-dependent apoptosis, but it inhibits apoptosis induced by the death receptor-mediated signaling pathway. Since GSK3 has two isoforms ( $\alpha$ and $\beta$ ), it is currently unclear whether (a) both isoforms have the same regulatory activities on apoptosis or (b) each of the isoforms has its unique regulatory role in apoptosis. Further characterization of fission yeast Skp1, its mammalian counterparts, and their role in Vpr-induced apoptosis will certainly shed light on the important regulatory mechanisms of Skp1/GSK3 in apoptosis.

Combining the results provided in this study with those from other previous studies, we would like to suggest fission yeast as a simple and reasonable model organism for studying pro- or anti-apoptotic functions of various apoptosis-inducing agents like HIV-1 Vpr. This proposition is based on the evidence illustrated here that Vpr-induced cell death in fission yeast resembles many aspects of the apoptotic processes in mammalian cells. More importantly, we have demonstrated that anti-apoptotic proteins, such as Ef2 and Hsp16, also suppress Vpr-induced cell death in fission yeast.

\section{Materials and Methods}

\section{Yeast strains, plasmids, and media}

All of the fission yeast strains used in this study are wild-type S. pombe strain SP223 (h-ade6-216 leu1-32 ura4-294) and its derivatives that have been reported previously. The MZ01 strain is SP223 transformed with pYZ1N-vpr [33, 41]. The RE007 strain (h-ade6-216 leu1-32 ura4-294::vpr(NL4-3)::ura4+) carries a single integrated copy of the $v p r$ gene under the $n m t 1$ promoter in the chromosome of SP223 [28]. Details of the Vpr mutant panel have been described previously [33].

\section{Cell growth and vpr gene induction in fission yeast cells}

All of the fission yeast strains were grown in the EMM using standard culture techniques [70]. The vpr gene was expressed under an $n m t 1$ (no message in thiamine) promoter as previously described $[32,71]$. Cells carrying plasmids with the $n m t 1$ promoter were maintained selectively in appropriately supplemented EMM with $20 \mu \mathrm{M}$ thiamine. For gene induction, cells were first grown to the mid-log growth phase in the presence of $20 \mu \mathrm{M}$ thiamine. Cells were then washed three times with distilled water and diluted to a final concentration of approximately $2 \times 10^{5}$ cells $/ \mathrm{ml}$ in $5 \mathrm{ml}$ of appropriately supplemented EMM with (gene-off) or without (geneon) thiamine. All cells were normally grown at $30^{\circ} \mathrm{C}$ with constant shaking at $200 \mathrm{rpm}$.

\section{Measurement of Vpr-induced cell death by colony-forming ability \\ Colony-forming ability was used as a quantitative measurement of Vpr-induced cell death as previously described [27, 33]. Briefly, $S$. pombe cells containing $v p r$ gene constructs were grown to the mid-log growth phase in the presence of $20 \mu \mathrm{M}$ thiamine and washed three times with distilled water. Equal numbers of cells were plated}


onto selective EMM plates with or without thiamine. Agar plates were incubated at $30{ }^{\circ} \mathrm{C}$ for 3-4 days to obtain individual colonies. Colony-forming units (CFU) of vpr-expressing cells were calculated from the number of colonies that grew onto thiamine-free EMM plates as a percentage of the number of cells originally plated, corrected by the plating efficiency of $v p r$-repressing cells. The plating efficiency of $v p r$-repressing cells ranged from $40 \%$ to $100 \%$ and was determined as the number of colonies obtained after plating a defined number of cells onto thiamine-containing EMM plates. Cell survival of $v p r$-expressing cells was quantified by using the same method described above, with the exception that $v p r$-expressing cells were plated $24 \mathrm{~h}$ after gene induction.

\section{Annexin V staining}

Annexin V staining was performed using the ApoAlert Annexin V Apoptosis kit (Clontech) as previously described [20, 22]. Briefly, vprexpressing and $v p r$-repressing cells were washed in sorbitol buffer (1.2 $\mathrm{M}$ sorbitol, $0.5 \mathrm{mM} \mathrm{MgCl}_{2}$, and $35 \mathrm{mM}$ potassium phosphate, $\mathrm{pH}$ 6.8) and treated with zymolyase for $2 \mathrm{~h}$ at room temperature. Spheroplasts were then washed and resuspended in binding buffer (1.2 M sorbitol, $10 \mathrm{mM}$ HEPES/NaOH, $140 \mathrm{mM} \mathrm{NaCl}$, and $2.5 \mathrm{mM}$ $\mathrm{CaCl}_{2}$ ). Staining with fluorescein-conjugated Annexin V and PI was performed simultaneously. A total of $2 \mu \mathrm{l}$ of Annexin V-FITC and $2 \mu \mathrm{l}$ of PI were added to $38 \mu \mathrm{l}$ of cell suspension and then incubated for $20 \mathrm{~min}$ at room temperature. The cells were harvested 32 and $48 \mathrm{~h}$ after gene induction, resuspended in binding buffer, and applied to microscope slides. The PI was used here as a control to ensure the integrity of the plasma membrane, since PI normally cannot pass through an intact plasma membrane [22].

\section{Visualization of mitochondria and assessment of mitochon- drial potential}

Mitochondria in S. pombe were visualized microscopically using the vital dye DASPMI (Sigma) as previously described [43, 72]. DASPMI was added to the culture at a final concentration of $75 \mu \mathrm{g} / \mathrm{ml}$, and cells were incubated at $36{ }^{\circ} \mathrm{C}$ for $5 \mathrm{~min}$. Cells were then harvested $24 \mathrm{~h}$ after gene induction, resuspended in EMM, and examined immediately with the fluorescence microscope. To assess mitochondrial potential $\left(\triangle \psi_{\mathrm{m}}\right), v p r$-expressing and vpr-repressing cells were treated with $\mathrm{DiOC}_{6}(3)$ at a final concentration of $40 \mathrm{nM}$ at $30{ }^{\circ} \mathrm{C}$ for $10 \mathrm{~min}$ and then subjected to Becton Dickinson FACScan analysis [63].

\section{Fluorescence microscopy}

A Leica DMR fluorescence microscope (DM4500B; Leica Microsystems) equipped with a high-performance camera (Hamamatsu) and OpenLab software (Improvision) was used for all imaging analyses. Living cells were observed under the microscope, and images were captured at various time points after $v p r$ gene induction as indicated. The production of ROS was detected with the ROS indicator dye DHE (Sigma) or $\mathrm{H}_{2}$ DCFDA (Invitrogen) [22, 24, 73]. DHE was added to the culture at a final concentration of $5 \mu \mathrm{g} / \mathrm{ml}$ after $20.5 \mathrm{~h}$ of $v p r$ induction. As an alternative procedure, $\mathrm{H}_{2}$ DCFDA was added to the culture at a final concentration of $10 \mu \mathrm{g} / \mathrm{ml}$ after $22.5 \mathrm{~h}$ of $v p r$ gene induction. Cells were also treated with the free radical spin trap reagent TMPO (Alexis Biochemicals) at a final concentration of $125 \mu \mathrm{g} / \mathrm{ml}$, and cells were visualized for ROS production by using DHE $[22,24]$. TMPO was added twice during the course of $v p r$ expression. TMPO was first added to the culture when the cells were diluted to start $v p r$ induction, and cells were treated again with TMPO when DHE was added to the culture [22].

\section{Acknowledgments}

This study was supported in part by grants from the National Institute of Health (USA) AI40891 and GM63080 (Richard Y Zhao). The content described in this manuscript is covered under a provisional patent application No. U.S. $60 / 698,624$.

\section{References}

1 Varbanov M, Espert L, Biard-Piechaczyk M. Mechanisms of CD4 T-cell depletion triggered by HIV-1 viral proteins. AIDS Rev 2006; 8:221-236.

2 Petit F, Arnoult D, Viollet L, Estaquier J. Intrinsic and extrinsic pathways signaling during HIV-1 mediated cell death. Biochimie 2003; 85:795-811.

3 Gougeon ML, Montagnier L. Programmed cell death as a mechanism of CD4 and CD8T cell deletion in AIDS. Molecular control and effect of highly active anti-retroviral therapy. Ann N Y Acad Sci 1999; 887:199-212.

4 Andersen JL, Dehart JL, Zimmerman ES, et al. HIV-1 Vpr-induced apoptosis is cell cycle dependent and requires Bax but not ANT. PLoS Pathog 2006; 2:e127.

5 Andersen JL, Zimmerman ES, DeHart JL, et al. ATR and GADD45alpha mediate HIV-1 Vpr-induced apoptosis. Cell Death Differ 2005; 12:326-334.

6 Jacotot E, Ferri K, El Hamel C, et al. Control of mitochondrial membrane permeabilization by adenine nucleotide translocator interacting with HIV-1 viral protein $\mathrm{r}$ and bcl-2. J Exp Med 2001; 193:509-520.

7 Jacotot E, Ravagnan L, Loeffler M, et al. The HIV-1 viral protein $\mathrm{R}$ induces apoptosis via a direct effect on the mitochondrial permeability transition pore. J Exp Med 2000; 191:33-46.

8 Stewart SA, Poon B, Jowett JB, Chen IS. Human immunodeficiency virus type $1 \mathrm{Vpr}$ induces apoptosis following cell cycle arrest. J Virol 1997; 71:5579-5592.

9 Zhao Y, Elder RT. Yeast perspectives on HIV-1 Vpr. Front Biosci 2000; 5:D905-D916.

10 Muthumani K, Choo AY, Hwang DS, et al. Mechanism of HIV-1 viral protein R-induced apoptosis. Biochem Biophys Res Commun 2003; 304:583-592.

11 Green DR, Kroemer G. The pathophysiology of mitochondrial cell death. Science 2004; 305:626-629.

12 Brenner C, Kroemer G. The mitochondriotoxic domain of Vpr determines HIV-1 virulence. J Clin Invest 2003; 111:1455-1457.

13 Muthumani K, Hwang DS, Desai BM, et al. HIV-1 Vpr induces apoptosis through caspase 9 in $\mathrm{T}$ cells and peripheral blood mononuclear cells. J Biol Chem 2002; 277:37820-37831.

14 Muthumani K, Zhang D, Hwang DS, et al. Adenovirus encoding HIV-1 Vpr activates caspase 9 and induces apoptotic cell death in both $\mathrm{p} 53$ positive and negative human tumor cell lines. Oncogene 2002; 21:4613-4625.

15 Stewart SA, Poon B, Song JY, Chen IS. Human immunodeficiency virus type $1 \mathrm{vpr}$ induces apoptosis through caspase activation. J Virol 2000; 74:3105-3111. 
16 Lum JJ, Cohen OJ, Nie Z, et al. Vpr R77Q is associated with long-term nonprogressive HIV infection and impaired induction of apoptosis. J Clin Invest 2003; 111:1547-1554.

17 Patel CA, Mukhtar M, Pomerantz RJ. Human immunodeficiency virus type 1 Vpr induces apoptosis in human neuronal cells. $J$ Virol 2000; 74:9717-9726.

18 Roumier T, Vieira HL, Castedo M, et al. The C-terminal moiety of HIV-1 Vpr induces cell death via a caspase-independent mitochondrial pathway. Cell Death Differ 2002; 9:1212-1219.

19 Varadarajan $\mathrm{P}$, Mahalingam S, Liu P, et al. The functionally conserved nucleoporins Nup124p from fission yeast and the human Nup153 mediate nuclear import and activity of the Tf1 retrotransposon and HIV-1 Vpr. Mol Biol Cell 2005; 16:18231838.

20 Madeo F, Frohlich E, Frohlich KU. A yeast mutant showing diagnostic markers of early and late apoptosis. J Cell Biol 1997; 139:729-734.

21 Frohlich KU, Madeo F. Apoptosis in yeast - a monocellular organism exhibits altruistic behaviour. FEBS Lett 2000; 473:6-9.

22 Zhang Q, Chieu HK, Low CP, et al. Schizosaccharomyces pombe cells deficient in triacylglycerols synthesis undergo apoptosis upon entry into the stationary phase. J Biol Chem 2003; 278:47145-47155.

23 Madeo F, Herker E, Maldener C, et al. A caspase-related protease regulates apoptosis in yeast. Mol Cell 2002; 9:911-917.

24 Madeo F, Frohlich E, Ligr M, et al. Oxygen stress: a regulator of apoptosis in yeast. J Cell Biol 1999; 145:757-767.

25 Low CP, Liew LP, Pervaiz S, Yang H. Apoptosis and lipoapoptosis in the fission yeast Schizosaccharomyces pombe. FEMS Yeast Res 2005; 5:1199-1206.

26 Jowett JB, Planelles V, Poon B, et al. The human immunodeficiency virus type $1 \mathrm{vpr}$ gene arrests infected T cells in the G2 + M phase of the cell cycle. J Virol 1995; 69:6304-6313.

27 Zhao Y, Yu M, Chen M, et al. Pleiotropic effects of HIV-1 protein $\mathrm{R}$ (Vpr) on morphogenesis and cell survival in fission yeast and antagonism by pentoxifylline. Virol 1998; 246:266-276.

28 Benko Z, Liang D, Agbottah E, et al. Anti-Vpr activity of a yeast chaperone protein. J Virol 2004; 78:11016-11029.

29 Benko Z, Liang D, Agbottah E, et al. Antagonistic interaction of HIV-1 Vpr with Hsf-mediated cellular heat shock response and Hsp16 in fission yeast (Schizosaccharomyces pombe). Retrovirology $2007 ; 4: 16$.

30 Iordanskiy S, Zhao Y, Dubrovsky L, et al. Heat shock protein 70 protects cells from cell cycle arrest and apoptosis induced by human immunodeficiency virus type 1 viral protein R. J Virol 2004; 78:9697-9704.

31 Zelivianski S, Liang D, Chen M, Mirkin BL, Zhao RY. Suppressive effect of elongation factor 2 on apoptosis induced by HIV-1 viral protein R. Apoptosis 2006; 11:377-388.

32 Zhao Y, Cao J, O Gorman MRG, Yu M, Yogev R. Effect of human immunodeficiency virus Type 1 protein $\mathrm{R}$ (vpr) gene expression on basic cellular functions of fission yeast Schizosaccharomyces pombe. J Virol 1996; 70:5821-5826.

33 Chen M, Elder RT, Yu M, et al. Mutational analysis of Vpr-induced G2 arrest, nuclear localization, and cell death in fission yeast. J Virol 1999; 73:3236-3245.

34 Vance JE, Steenbergen R. Metabolism and functions of phosphatidylserine. Prog Lipid Res 2005; 44:207-234.

35 Hockenbery DM, Oltvai ZN, Yin XM, Milliman CL, Korsmeyer
SJ. Bcl-2 functions in an antioxidant pathway to prevent apoptosis. Cell 1993; 75:241-251.

36 Kane DJ, Sarafian TA, Anton R, et al. Bcl-2 inhibition of neural death: decreased generation of reactive oxygen species. Science 1993; 262:1274-1277.

37 Fleury C, Mignotte B, Vayssiere JL. Mitochondrial reactive oxygen species in cell death signaling. Biochimie 2002; 84:131141.

38 Orrenius S, Gogvadze V, Zhivotovsky B. Mitochondrial oxidative stress: implications for cell death. Annu Rev Pharmacol Toxicol 2007; 47:143-183.

39 Hardy M, Rockenbauer A, Vasquez-Vivar J, et al. Detection, characterization, and decay kinetics of ROS and thiyl adducts of mito-DEPMPO spin trap. Chem Res Toxicol 2007; 20:10531060.

40 Petit P, Glab N, Marie D, Kieffer H, Metezeau P. Discrimination of respiratory dysfunction in yeast mutants by confocal microscopy, image, and flow cytometry. Cytometry 1996; 23:28-38.

41 Zhao Y, Elder RT, Chen M, Cao J. Fission yeast expression vectors adapted for positive identification of gene insertion and green fluorescent protein fusion. BioTechniques 1998; 25:438440, 442, 444.

42 Miyakawa I, Aoi H, Sando N, Kuroiwa T. Fluorescence microscopic studies of mitochondrial nucleoids during meiosis and sporulation in the yeast, Saccharomyces cerevisiae. J Cell Sci 1984; 66:21-38.

43 Yaffe MP, Harata D, Verde F, et al. Microtubules mediate mitochondrial distribution in fission yeast. Proc Natl Acad Sci USA 1996; 93:11664-11668.

44 Schuler W, Wecker K, de Rocquigny H, et al. NMR structure of the (52-96) C-terminal domain of the HIV-1 regulatory protein Vpr: molecular insights into its biological functions. J Mol Biol 1999; 285:2105-2117.

45 Wecker K, Roques BP. NMR structure of the (1-51) N-terminal domain of the HIV-1 regulatory protein Vpr. Eur J Biochem 1999; 266:359-369.

46 Ludovico P, Sousa MJ, Silva MT, Leao C, Corte-Real M. Saccharomyces cerevisiae commits to a programmed cell death process in response to acetic acid. Microbiology 2001; 147:2409-2415.

47 Reiter J, Herker E, Madeo F, Schmitt MJ. Viral killer toxins induce caspase-mediated apoptosis in yeast. J Cell Biol 2005; 168:353-358.

48 Hanada M, Aime-Sempe C, Sato T, Reed JC. Structure-function analysis of $\mathrm{Bcl}-2$ protein. Identification of conserved domains important for homodimerization with Bcl-2 and heterodimerization with Bax. J Biol Chem 1995; 270:11962-11969.

49 Jurgensmeier JM, Krajewski S, Armstrong RC, et al. Bax- and Bak-induced cell death in the fission yeast Schizosaccharomyces pombe. Mol Biol Cell 1997; 8:325-339.

50 Ligr M, Madeo F, Frohlich E, et al. Mammalian Bax triggers apoptotic changes in yeast. FEBS Lett 1998; 438:61-65.

51 Torgler CN, de Tiani M, Raven T, et al. Expression of bak in $S$. pombe results in a lethality mediated through interaction with the calnexin homologue Cnx1. Cell Death Differ 1997; 4:263-271.

52 Ludovico P, Rodrigues F, Almeida A, et al. Cytochrome c release and mitochondria involvement in programmed cell death induced by acetic acid in Saccharomyces cerevisiae. Mol Biol Cell 2002; 13:2598-2606.

53 Manon S, Chaudhuri B, Guerin M. Release of cytochrome c and 
decrease of cytochrome c oxidase in Bax-expressing yeast cells, and prevention of these effects by coexpression of Bcl-xL. FEBS Lett 1997; 415:29-32.

54 Deniaud A, Brenner C, Kroemer G. Mitochondrial membrane permeabilization by HIV-1 Vpr. Mitochondrion 2004; 4:223233.

55 Macreadie IG, Thorburn DR, Kirby DM, et al. HIV-1 protein Vpr causes gross mitochondrial dysfunction in the yeast Saccharomyces cerevisiae. FEBS Lett 1997; 410:145-149.

56 Bossy-Wetzel E, Newmeyer DD, Green DR. Mitochondrial cytochrome c release in apoptosis occurs upstream of DEVDspecific caspase activation and independently of mitochondrial transmembrane depolarization. EMBO J 1998; 17:37-49.

57 Vander Heiden MG, Chandel NS, Williamson EK, Schumacker PT, Thompson CB. Bcl-xL regulates the membrane potential and volume homeostasis of mitochondria. Cell 1997; 91:627-637.

58 Matsuyama S, Llopis J, Deveraux QL, Tsien RY, Reed JC. Changes in intramitochondrial and cytosolic $\mathrm{pH}$ : early events that modulate caspase activation during apoptosis. Nat Cell Biol 2000; 2:318-325.

59 Krohn AJ, Wahlbrink T, Prehn JH. Mitochondrial depolarization is not required for neuronal apoptosis. JNeurosci 1999; 19:73947404.

60 Giovannini C, Matarrese P, Scazzocchio B, et al. Mitochondria hyperpolarization is an early event in oxidized low-density lipoprotein-induced apoptosis in Caco-2 intestinal cells. FEBS Lett 2002; 523:200-206.

61 Iijima T. Mitochondrial membrane potential and ischemic neuronal death. Neurosci Res 2006; 55:234-243.

62 England K, Cotter TG. Direct oxidative modifications of signalling proteins in mammalian cells and their effects on apoptosis. Redox Rep 2005; 10:237-245.

63 Gross A, Pilcher K, Blachly-Dyson E, et al. Biochemical and genetic analysis of the mitochondrial response of yeast to BAX and BCL-X(L). Mol Cell Biol 2000; 20:3125-3136.

64 Li PF, Dietz R, von Harsdorf R. p53 regulates mitochondrial membrane potential through reactive oxygen species and induces cytochrome c-independent apoptosis blocked by Bcl-2. EMBO $J$ 1999; 18:6027-6036.

65 Zamzami N, Kroemer G. Condensed matter in cell death. Nature 1999; 401:127-128.
66 Elder RT, Yu M, Chen M, Edelson S, Zhao Y. Cell cycle G2 arrest induced by HIV-1 Vpr in fission yeast (Schizosaccharomyces pombe) is independent of cell death and early genes in the DNA damage checkpoint. Viral Res 2000; 68:161-173.

67 Frame S, Cohen P. GSK3 takes centre stage more than 20 years after its discovery. Biochem J 2001; 359:1-16.

68 Jope RS, Johnson GV. The glamour and gloom of glycogen synthase kinase-3. Trends Biochem Sci 2004; 29:95-102.

69 Beurel E, Jope RS. The paradoxical pro- and anti-apoptotic actions of GSK3 in the intrinsic and extrinsic apoptosis signaling pathways. Prog Neurobiol 2006; 79:173-189.

70 Moreno S, Klar A, Nurse P. Molecular genetic analysis of fission yeast Schizosaccharomyces pombe. Methods Enzymol 1991; 194:795-823.

71 Maundrell K. Thiamine-repressible expression vectors $\mathrm{pREP}$ and pRIP for fission yeast. Gene 1993; 123:127-130.

72 Weir BA, Yaffe MP. Mmd1p, a novel, conserved protein essential for normal mitochondrial morphology and distribution in the fission yeast Schizosaccharomyces pombe. Mol Biol Cell 2004; 15:1656-1665.

73 Marchetti MA, Weinberger M, Murakami Y, Burhans WC, Huberman JA. Production of reactive oxygen species in response to replication stress and inappropriate mitosis in fission yeast. $J$ Cell Sci 2006; 119:124-131.

74 Elder RT, Yu M, Chen M, et al. HIV-1 Vpr induces cell cycle G2 arrest in fission yeast (Schizosaccharomyces pombe) through a pathway involving regulatory and catalytic subunits of PP2A and acting on both Wee1 and Cdc25. Virology 2001; 287:359-370.

75 Rothe G, Kellermann W, Valet G. Flow cytometric parameters of neutrophil function as early indicators of sepsis- or traumarelated pulmonary or cardiovascular organ failure. J Lab Clin Med 1990; 115:52-61.

76 Gomes A, Fernandes E, Lima JL. Fluorescence probes used for detection of reactive oxygen species. J Biochem Biophys Methods 2005; 65:45-80.

77 Selig L, Benichou S, Rogel ME, et al. Uracil DNA glycosylase specifically interacts with Vpr of both human immunodeficiency virus type 1 and simian immunodeficiency virus of sooty mangabeys, but binding does not correlate with cell cycle arrest. J Virol 1997; 71:4842-4846. 